\title{
Prospective associations between socio-economic status and dietary patterns in European children: the Identification and Prevention of Dietary- and Lifestyle-induced Health Effects in Children and Infants (IDEFICS) Study
}

\author{
Juan Miguel Fernández-Alvira ${ }^{1,2 *}$, Claudia Börnhorst ${ }^{2}$, Karin Bammann ${ }^{3}$, Wencke Gwozdz ${ }^{4}$, \\ Vittorio Krogh ${ }^{5}$, Antje Hebestreit ${ }^{2}$, Gianvincenzo Barba ${ }^{6}$, Lucia Reisch ${ }^{4}$, Gabriele Eiben ${ }^{7}$, Iris Iglesia ${ }^{1}$, \\ Tomas Veidebaum ${ }^{8}$, Yannis A. Kourides ${ }^{9}$, Eva Kovacs ${ }^{10}$, Inge Huybrechts ${ }^{11,12}$, Iris Pigeot ${ }^{2,13}$ and \\ Luis A. Moreno ${ }^{1}$ on behalf of the IDEFICS consortium \\ ${ }^{1}$ GENUD (Growth, Exercise, NUtrition and Development) Research Group, Faculty of Health Sciences, University of Zaragoza, \\ Edificio del SAI, C/Pedro Cerbuna s/n, 50009 Zaragoza, Spain \\ ${ }^{2}$ Leibniz Institute for Prevention Research and Epidemiology - BIPS, Bremen, Germany \\ ${ }^{3}$ Institute for Public Health and Nursing Sciences (IPP), University of Bremen, Bremen, Germany \\ ${ }^{4}$ Copenhagen Business School, Copenhagen, Denmark \\ ${ }^{5}$ Epidemiology Unit, Department of Preventive and Predictive Medicine, Fondazione IRCCS Istituto Nazionale dei Tumori, \\ Milan, Italy \\ ${ }^{6}$ Unit of Epidemiology and Population Genetics, Institute of Food Sciences, National Research Council, Avellino, Italy \\ ${ }^{7}$ Public Health Epidemiology Unit (EPI), Department of Public Health and Community Medicine, Sablgrenska Academy, \\ University of Gothenburg, Gothenburg, Sweden \\ ${ }^{8}$ Department of Chronic Diseases, National Institute for Health Development, Tallinn, Estonia \\ ${ }^{9}$ Research and Education Institute of Child Health, Strovolos, Cyprus \\ ${ }^{10}$ Department of Paediatrics, University of Pécs, Pécs, Hungary \\ ${ }^{11}$ Department of Public Health, Ghent University, Ghent, Belgium \\ ${ }^{12}$ International Agency for Research on Cancer (IARC), Dietary Exposure Assessment Group, Lyon, France \\ ${ }^{13}$ Faculty of Mathematics and Computer Science, Institute of Statistics, University Bremen, Bremen, Germany
}

(Submitted 3 April 2014 - Final revision received 24 September 2014 - Accepted 17 October 2014 - First published online 7 January 2015)

\section{Abstract}

Exploring changes in children's diet over time and the relationship between these changes and socio-economic status (SES) may help to understand the impact of social inequalities on dietary patterns. The aim of the present study was to describe dietary patterns by applying a cluster analysis to 9301 children participating in the baseline (2-9 years old) and follow-up (4-11 years old) surveys of the Identification and Prevention of Dietary- and Lifestyle-induced Health Effects in Children and Infants Study, and to describe the cluster memberships of these children over time and their association with SES. We applied the $K$-means clustering algorithm based on the similarities between the relative frequencies of consumption of forty-two food items. The following three consistent clusters were obtained at baseline and follow-up: processed (higher frequency of consumption of snacks and fast food); sweet (higher frequency of consumption of sweet foods and sweetened drinks); healthy (higher frequency of consumption of fruits, vegetables and wholemeal products). Children with higher-educated mothers and fathers and the highest household income were more likely to be allocated to the healthy cluster at baseline and follow-up and less likely to be allocated to the sweet cluster. Migrants were more likely to be allocated to the processed cluster at baseline and follow-up. Applying the cluster analysis to derive dietary patterns at the two time points allowed us to identify groups of children from a lower socio-economic background presenting persistently unhealthier dietary profiles. This finding reflects the need for healthy eating interventions specifically targeting children from lower socio-economic backgrounds.

Key words: Cluster analysis: Dietary behaviour: FFQ: Income: Maternal education: Paternal education

Abbreviations: CEHQ-FFQ, Children's Eating Habits Questionnaire-FFQ; IDEFICS, Identification and Prevention of Dietary- and Lifestyle-induced Health Effects in Children and Infants; PCA, principal components analysis; SES, socio-economic status; T0, baseline; T1, follow-up after the intervention.

*Corresponding author: J. M. Fernández-Alvira, fax +34 8765540 9, email juanfdez@unizar.es 
The influence of socio-economic status (SES) on health has been observed for all age groups. Due to the differences in health-related behaviours, health knowledge, housing conditions, psychosocial stressors, access to health care, etc., people living under lower socio-economic conditions have a heavier burden of disease compared with their better-off counterparts ${ }^{(1,2)}$. More specifically, diet quality and food consumption have been shown to be associated with several indicators of SES (e.g. income and educational attainment) and to factors leading to social vulnerability (e.g. migration), which, in turn, can affect overall health and increase the predisposition to developing certain disorders such as overweight and obesity ${ }^{(3,4)}$.

Previous studies focusing on the associations between indicators of SES and food intake in children and adolescents reported a lower intake of fruits and vegetables and a higher intake of energy-dense foods in lower-SES groups ${ }^{(5,6)}$. Other studies have focused on dietary patterns instead and their associations with indicators of $\mathrm{SES}^{(7,8)}$. Indeed, considering diet as a whole is of great relevance for describing groups at a higher risk of developing overweight and obesity, as the overall diet seems to be a more important determinant of weight gain compared with single dietary components $^{(9,10)}$

Dietary pattern analysis has been increasingly applied in recent years to assess the relationship between overall diet and the risk of chronic diseases ${ }^{(10)}$. Cluster analysis, a commonly applied method to derive dietary patterns, clusters individuals into non-overlapping groups that reflect relatively homogeneous dietary patterns within groups and distinct dietary patterns between groups. Various studies have applied this method to derive dietary patterns in children and adolescents and explored their associations with indicators of $\mathrm{SES}^{(11-13)}$. Moreover, exploring the changes in children's diet over time and the relationship between these changes and indicators of SES may help to identify the changes in dietary patterns and/or children changing their dietary patterns, thus allowing a better understanding of the impact of social inequalities on diet. Changes in diet over time have been previously explored using the principal components analysis (PCA); however, to the best of our knowledge, there is as yet only one report examining children's dietary patterns over time using cluster analysis ${ }^{(14)}$. The PCA provides linear combinations of foods instead of referring to identifiable groups of individuals, while cluster analysis identifies relatively homogeneous groups of children based on their food consumption. Applying cluster analysis to describe longitudinal changes in dietary patterns can provide further insight into changes in children's dietary patterns and the identification of groups with persistently unhealthier diets.

Therefore, the primary aim of the present study was to describe dietary patterns by applying cluster analysis to children participating in the baseline and follow-up surveys of the Identification and Prevention of Dietaryand Lifestyle-induced Health Effects in Children and Infants (IDEFICS) Study. The secondary aim was to describe the cluster memberships of children over time and their associations with SES.

\section{Subjects and methods}

The IDEFICS Study is a multi-centre, population-based study of children aged 2-9 years upon recruitment in selected regions of eight European countries (Belgium, Cyprus, Estonia, Germany, Hungary, Italy, Spain and Sweden) ${ }^{(15,16)}$. Each participating country included one intervention region, where the community intervention programme took place, and an equivalent control region ${ }^{(17)}$. The present study conducted two main surveys (baseline (T0) and follow-up after the intervention (T1)) in pre-schools and primary school classes (first and second grades at baseline). The baseline survey (September 2007 to May 2008) included 16228 children aged 2-9 years (median age 6.3 (range 7.7) years). The follow-up survey (September 2009 to May 2010) reached an overall response rate of $68 \%$ and included 11038 children aged 4-11 years. The general design of the IDEFICS Study has been described elsewhere ${ }^{(15,16)}$. The present study includes only children with $<50 \%$ of missing values in FFQ data at T0 and T1 and for whom information on socio-economic variables and anthropometric measures was available (n 9301 children; 50.3\% boys; Fig. 1). Each participating centre obtained ethical approval from its health research ethics authority. All children provided oral consent and their parents provided written informed consent for all examinations and for the collection of samples, analysis and storage of personal data and collected samples.

\section{Measurements}

Dietary data were obtained at both T0 and T1 using the food frequency section of the Children's Eating Habits Questionnaire-FFQ (CEHQ-FFQ) ${ }^{(18)}$, a validated screening tool in which the frequency of the child's consumption of selected food items during the preceding 4 weeks was reported by the parents. In order to assess meals under parental control, the questionnaire referred to meals outside the school canteen or childcare meal provision settings only ${ }^{(18,19)}$. The CEHQFFQ, which consists of forty-three food items clustered into fourteen food groups, was applied as a screening instrument to investigate the consumption of foods shown to be related, either positively or negatively, to overweight and obesity in children. The CEHQ-FFQ was not designed to provide

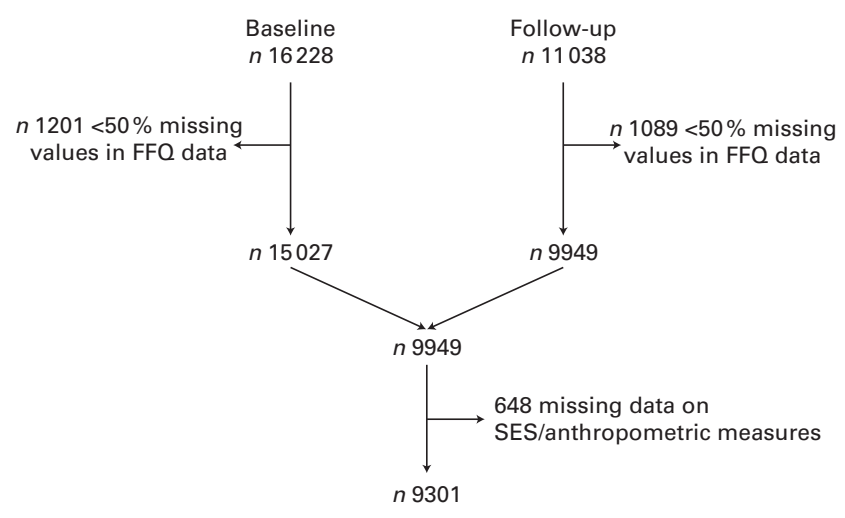

Fig. 1. Selection of the final study sample. SES, socio-economic status. 
an estimate of total energy intake or total food intake ${ }^{(19)}$. Response options displayed from left to right were as follows: never/less than once per week; one to three times per week; four to six times per week; one time per d; two times per d; three times per $\mathrm{d}$; four or more times per $\mathrm{d}$; I have no idea. For the analysis of dietary patterns, a conversion factor was used to transform the answers in the questionnaire into weekly consumption frequencies, represented by a number ranging from 0 to 30 . Only children with $<50 \%$ of missing values and with valid data on anthropometric measures and socio-economic variables were included in the analyses ${ }^{(20)}$. Multiple imputation was applied using sex, age, BMI and country as predictors for the remaining missing values (median number of available items 43 (SD 2.55)) ${ }^{(21)}$.

During the baseline and follow-up surveys, parents completed a self-administered questionnaire on parental attitudes, children's behaviour and social environment. Parental education and income were self-reported. Parental education level was categorised according to the International Standard Classification of Education- $97^{(22)}$. Household income was assessed using nine country-specific categories based on the median equivalent income. The gained amount was then equalised to the number of household members using the square root scale provided by the Organisation for Economic Co-operation and Development ${ }^{(23)}$. Additionally, migrant background was assessed. A migrant background was assumed if one or both of the parents were born in another country.

Trained staff carried out anthropometric measurements at T0 and T1 following a standardised procedure. Body height (cm) was measured without shoes and all plaits undone using a portable stadiometer (SECA 225). Weight (kg) was measured using a child-adapted version of the electronic scale Tanita BC 420 SMA with the children in the fasting state $(>8 \mathrm{~h}$ since the last meal) and wearing only underwear $^{(24)}$. BMI and age- and sex-specific BMI $z$-scores were calculated and categorised according to the criteria proposed by the International Obesity Task Force ${ }^{(25)}$.

\section{Statistical analyses}

$K$-means cluster analysis was performed to identify clusters of children with similar dietary patterns ${ }^{(26)}$. First, all the variables in the FFQ were checked for their suitability in cluster analysis in terms of relevance. The item 'meat replacement products' was not included in the set of variables as more than $95 \%$ of the subjects reported 'never/less than once per week' as the frequency of consumption. Second, correlations between single food items were checked to assess multi-collinearity. The assessment of their correlations showed no redundant variables. Therefore, all the remaining (forty-two) food items were taken into account. The relative frequency of consumption was calculated for each food item by dividing the frequency of consumption of a specific food item by the sum of the consumption frequencies of all the food items reported for each single subject. The $z$-scores of the relative consumption frequency were calculated to standardise the dataset before clustering, as differences in variances of the variables may otherwise affect the resulting clusters ${ }^{(27)}$. A positive value indicates a higher frequency of consumption and a negative value reflects a lower frequency of consumption. The $K$-means algorithm was applied with a pre-defined maximum of 100 iterations to generate separate cluster solutions for two to six clusters. In order to find a stable clustering pattern, several solutions were obtained with different starting seeds. Iterations were generated until no change in cluster centroids was observed. The stability of the final solution was examined by randomly splitting the database into half and repeating the same clustering procedure, until satisfactory results were observed (a maximum of 327 children in the baseline clustering and 495 children in the follow-up clustering being allocated to different clusters, representing 3.5 and $5.3 \%$ of the total sample, respectively). This procedure was applied for both baseline and follow-up datasets.

The stability of the cluster solutions and the interpretability of the clusters were considered as the criteria for choosing the final number of clusters to be retained. The clusters were labelled based on the $z$-scores of the food items.

Distribution of children in different clusters was calculated, stratified by sex, age, BMI status and country, both at T0 and T1. To assess the changes in dietary patterns over time, the cluster memberships of children at T0 and T1 were cross-tabulated, showing the proportion of children being allocated to the same or different clusters. Based on logistic regression models, OR for being allocated to the same cluster at T0 and T1 (i.e. healthy, sweet or processed at both time points; three models) or for changing the cluster (processed/sweet to healthy or vice versa; two models) were calculated, where the alternative category consisted of all the remaining combinations of cluster memberships in each model. Sex, age group, BMI status, migrant status, maternal and paternal education level, household income, country, and a dummy variable indicating intervention $v$. control region were assessed at both time points and included as covariates in all the models. The significance level was set at $P \leq 0 \cdot 05$. The analyses were performed using the Statistical Package for the Social Sciences (version 20.0; SPSS, Inc.).

\section{Results}

Based on the forty-two food items and their relative frequency of consumption, the three cluster solutions were considered the most interpretable and stable for both baseline and follow-up datasets and therefore were retained. The following labels were assigned to the three clusters: processed ( $n 4427$ at T0, $n 2554$ at T1); sweet ( $n 1910$ at T0, $n 1939$ at T1); healthy ( $n 2964$ at T0, $n 4808$ at T1). Tables 1 and 2 present the mean $z$-scores and standard deviations of all the food items in the three clusters at T0 and T1. Dietary data for both surveys were more likely to be available for children with lower-educated parents and lower household income and for children with lower BMI compared with the complete IDEFICS Study samples (data not shown). The cluster solutions obtained were similar in terms of interpretability at both time points. The mean values of the majority of the food items differed markedly between the three clusters (Tables 1 and 2). 
Table 1. $z$-Scores of relative consumption frequencies in the three clusters at baseline

(Mean values and standard deviations)

\begin{tabular}{|c|c|c|c|c|c|c|}
\hline \multirow[b]{2}{*}{ Food items } & \multicolumn{2}{|c|}{ Processed ( $n$ 4427) } & \multicolumn{2}{|c|}{ Sweet $(n$ 1910) } & \multicolumn{2}{|c|}{ Healthy $(n$ 2964) } \\
\hline & Mean & SD & Mean & SD & Mean & SD \\
\hline Cooked vegetables, potatoes, beans & $-0.36^{\mathrm{c} *}$ & 0.74 & $0.42^{\mathrm{a}}+$ & 1.06 & $0.27^{\mathrm{b}}$ & $1 \cdot 10$ \\
\hline Fried potatoes, potato croquettes & $0.21^{\mathrm{a}}+$ & 1.13 & $0.08^{\mathrm{b}}$ & 0.95 & $-0.37^{c *}$ & 0.67 \\
\hline Raw vegetables & $-0.32^{b}$ & 0.72 & $-0.35^{\mathrm{b} *}$ & 0.73 & $0.70^{\mathrm{a}} \dagger$ & $1 \cdot 15$ \\
\hline Fresh fruits without added sugar & $-0.24^{b}$ & 0.88 & $-0 \cdot 24^{b *}$ & 0.81 & $0.51^{\mathrm{a}} \dagger$ & 1.09 \\
\hline Fresh fruits with added sugar & $0.23^{\mathrm{a}} \dagger$ & 1.19 & $-0.17^{\mathrm{b}}$ & 0.73 & $-0.24^{b *}$ & 0.73 \\
\hline Water & $0.21^{\mathrm{a}}+$ & 0.98 & $-0.49^{\mathrm{c} *}$ & 0.99 & $0.00^{\mathrm{b}}$ & 0.92 \\
\hline Fruit juices & $0.08^{\mathrm{b}}$ & 1.02 & $0.21^{\mathrm{a}}+$ & 1.15 & $-0.26^{c *}$ & 0.79 \\
\hline Sweetened drinks & $-0 \cdot 10^{b}$ & 0.76 & $0.58^{\mathrm{a}}+$ & 1.64 & $-0.22^{\mathrm{c} *}$ & 0.50 \\
\hline Diet drinks & $-0.14^{b}$ & 0.45 & $0.59^{\mathrm{a}} \dagger$ & 1.95 & $-0.17^{b *}$ & 0.33 \\
\hline Breakfast cereals, muesli, sweetened & $0.15^{\mathrm{a}} \dagger$ & 1.09 & $0.10^{\mathrm{a}}$ & 1.01 & $-0.29^{b *}$ & 0.76 \\
\hline Porridge, oat meal, gruel, cereals, muesli, unsweetened & $-0 \cdot 21^{b}$ & 0.72 & $-0.40^{\mathrm{c*}}$ & 0.49 & $0.57^{\mathrm{a}}+$ & 1.31 \\
\hline Plain unsweetened milk & $-0.22^{b}$ & 0.90 & $-0.23^{b *}$ & 0.83 & $0.48^{\mathrm{a}}+$ & 1.07 \\
\hline Sweetened milk & $0.29^{\mathrm{a}}+$ & $1 \cdot 13$ & $-0.02^{b}$ & 0.94 & $-0.42^{\mathrm{c*}}$ & 0.60 \\
\hline Plain unsweetened yogurt or kefir & $-0.08^{b}$ & 0.85 & $-0.29^{\mathrm{c} *}$ & 0.66 & $0.31^{\mathrm{a}}+$ & 1.27 \\
\hline Sweet yogurt, fermented milk beverages & $-0.01^{b}$ & 1.02 & $0.16^{\mathrm{a}}+$ & 1.02 & $-0.09^{\mathrm{c} *}$ & 0.94 \\
\hline Fresh or frozen fish, not fried & $0.02^{b}$ & 1.00 & $-0.33^{c}$ & 0.80 & $0 \cdot 19^{a} \dagger$ & 1.07 \\
\hline Fried fish, fish fingers & $0.03^{\mathrm{a}}+$ & 1.02 & $-0.06^{b}$ & 0.94 & $-0.01^{\mathrm{a}}$ & 1.00 \\
\hline Cold cuts, preserved, ready-to-cook meat products & $-0.04^{b}$ & 0.87 & $0.60^{\mathrm{a}}+$ & $1 \cdot 19$ & $-0.32^{\mathrm{c} *}$ & 0.86 \\
\hline Fresh meat, not fried & $0.21^{\mathrm{a}}+$ & 1.01 & $-0.30^{\mathrm{c} *}$ & 0.96 & $-0.12^{b}$ & 0.95 \\
\hline Fried meat & $-0.15^{\mathrm{c} \star}$ & 0.96 & $0.35^{\mathrm{a}}+$ & 1.09 & $0.00^{\mathrm{b}}$ & 0.95 \\
\hline Fried or scrambled eggs & $0 \cdot 25^{a}+$ & 1.09 & $-0 \cdot 26^{b *}$ & 0.82 & $-0.21^{b}$ & 0.85 \\
\hline Boiled or poached eggs & $0.07^{a}+$ & 1.27 & $-0.14^{\mathrm{c} *}$ & 0.72 & $-0.02^{b}$ & 0.62 \\
\hline Mayonnaise, mayonnaise-based products & $-0.04^{b}$ & 0.86 & $0.52^{\mathrm{a}}+$ & 1.53 & $-0.28^{\mathrm{c}}$ & 0.52 \\
\hline Cheese & $0.07^{\mathrm{a}}+$ & 1.05 & $-0.17^{c *}$ & 0.90 & $0.00^{\mathrm{b}}$ & 0.98 \\
\hline Jam, honey & $-0.08^{b_{*}}$ & 0.90 & $0.29^{\mathrm{a}}+$ & 1.24 & $-0.07^{b}$ & 0.94 \\
\hline Chocolate- or nut-based spreads & $-0.11^{b}$ & 0.72 & $0.95^{\mathrm{a}} \dagger$ & 1.44 & $-0.44^{\mathrm{c*}}$ & 0.48 \\
\hline Butter, margarine on bread & $-0.08^{\mathrm{b} *}$ & 0.88 & $0.03^{\mathrm{a}}$ & 1.09 & $0.10^{\mathrm{a}}+$ & 1.09 \\
\hline Reduced-fat products on bread & $-0.28^{\mathrm{c} \star}$ & 0.58 & $0 \cdot 13^{\mathrm{b}}$ & $1 \cdot 12$ & $0.34^{\mathrm{a}}+$ & 1.26 \\
\hline Ketchup & $-0.07^{\mathrm{c} *}$ & 1.00 & $0.11^{\mathrm{a}}+$ & 1.04 & $0.03^{b}$ & 0.97 \\
\hline White bread, white roll, white crispbread & $0.31^{\mathrm{a}}+$ & 1.06 & $-0.08^{b}$ & 0.96 & $-0.42^{\mathrm{c} *}$ & 0.74 \\
\hline Wholemeal bread, dark roll, dark crispbread & $-0.36^{\mathrm{c} \star}$ & 0.73 & $0.27^{b}$ & $1 \cdot 17$ & $0.37^{\mathrm{a}} \dagger$ & 1.04 \\
\hline Pasta, noodles, rice & $-0.03^{b}$ & 1.04 & $-0.27^{c *}$ & 0.72 & $0.22^{a}+$ & 1.04 \\
\hline Dish of milled cereals & $0.03^{\mathrm{b}}$ & 1.06 & $-0.22^{\mathrm{c} *}$ & 0.55 & $0 \cdot 10^{\mathrm{a}} \dagger$ & $1 \cdot 10$ \\
\hline Pizza as main dish & $0.23^{\mathrm{a}}+$ & 1.22 & $-0.12^{b}$ & 0.81 & $-0.27^{\mathrm{c} *}$ & 0.58 \\
\hline Hamburgers, hot dogs, kebabs, wraps, falafel & $0.32^{\mathrm{a}} \dagger$ & $1 \cdot 16$ & $-0.48^{\mathrm{c} *}$ & 0.52 & $-0.17^{b}$ & 0.79 \\
\hline Nuts, seeds, dried fruits & $0.01^{\mathrm{b}}$ & 0.95 & $-0.27^{c *}$ & 0.69 & $0 \cdot 16^{a}+$ & 1.19 \\
\hline Crisps, maize (corn) crisps, popcorn & $0.20^{\mathrm{a}}+$ & $1 \cdot 13$ & $-0.04 b$ & 0.92 & $-0.26^{\mathrm{c} *}$ & 0.74 \\
\hline Savoury pastries, fritters & $0.37^{\mathrm{a}}+$ & $1 \cdot 20$ & $-0.38^{b *}$ & 0.55 & $-0.31^{b}$ & 0.62 \\
\hline Chocolate, candy bars & $0.19^{a}+$ & $1 \cdot 10$ & $0.13^{\mathrm{a}}$ & $1 \cdot 10$ & $-0.37^{b *}$ & 0.60 \\
\hline Candies, loose candies, marshmallows & $-0 \cdot 17^{b}$ & 0.78 & $0.72^{\mathrm{a}} \dagger$ & 1.48 & $-0.20^{b_{*}}$ & 0.63 \\
\hline Biscuits, packaged cakes, pastries, puddings & $-0.15^{\mathrm{b}}$ & 0.86 & $0.70^{a}+$ & $1 \cdot 30$ & $-0.24^{\mathrm{c} *}$ & 0.73 \\
\hline Ice cream, milk- or fruit-based bars & $0 \cdot 12^{\mathrm{a}} \dagger$ & $1 \cdot 14$ & $-0.16^{c_{*}}$ & 0.92 & $-0.07^{b}$ & 0.78 \\
\hline
\end{tabular}

a,b,c Mean values within a row with unlike superscript letters were significantly different $(P<0.05)$.

* The lowest mean value within a row.

†The highest mean value within a row.

Compared with the other clusters, the processed cluster presented at both time points had higher relative frequencies of consumption of takeaway and high-fat foods, such as savoury pastries and fritters; pizza as main dish; fried potatoes; hamburgers, hot dogs, kebabs and wraps; and crisps, maize (corn) crisps and popcorn. Products such as wholemeal bread, cooked vegetables, raw vegetables, and fresh fruits without added sugar scored lowest. At both time points, the sweet cluster had higher values for sugar-rich products, such as chocolateor nut-based spreads; sweetened drinks; fruit juices; diet drinks; candies, loose candies and marshmallows; and biscuits, packaged cakes, pastries and puddings, and had the lowest scores for water; porridge, oat meal, gruel, cereals and muesli, unsweetened; raw vegetables; plain unsweetened milk; and plain unsweetened yogurt and kefir. The healthy cluster had at both time points higher values for low-fat foods, foods rich in vitamins and whole-grain foods, e.g. raw vegetables; fresh fruits without added sugar; porridge, oat meal, gruel, cereals and muesli, unsweetened; and plain unsweetened milk, and lower values for high-fat and high-sugar products, such as fried potatoes; sweetened drinks; sweetened milk; mayonnaise and mayonnaise-based products; chocolate- or nut-based spreads; crisps, maize (corn) crisps and popcorn; and biscuits, packaged cakes, pastries and puddings.

Table 3 summarises the distribution of age, sex, BMI status and country in the three clusters at $\mathrm{T} 0$ and $\mathrm{T} 1$. The percentage of girls in the healthy cluster was slightly higher than that in the other two clusters, while a higher percentage of boys were allocated to the processed and sweet clusters. Older children represented a higher percentage in the processed and sweet clusters 
Table 2. $z$-Scores of relative consumption frequencies in the three clusters at follow-up (Mean values and standard deviations)

\begin{tabular}{|c|c|c|c|c|c|c|}
\hline \multirow[b]{2}{*}{ Food items } & \multicolumn{2}{|c|}{ Processed ( $n$ 2554) } & \multicolumn{2}{|c|}{ Sweet (n 1939) } & \multicolumn{2}{|c|}{ Healthy ( $n$ 4808) } \\
\hline & Mean & SD & Mean & SD & Mean & SD \\
\hline Cooked vegetables, potatoes, beans & $-0.45^{\mathrm{c} *}$ & 0.64 & $0.31^{a}+$ & $1 \cdot 12$ & $0 \cdot 11^{\mathrm{b}}$ & 1.02 \\
\hline Fried potatoes, potato croquettes & $0.42^{\mathrm{a}} \dagger$ & $1 \cdot 22$ & $0.16^{\mathrm{b}}$ & 1.01 & $-0 \cdot 29^{\mathrm{c} *}$ & 0.74 \\
\hline Raw vegetables & $-0.40^{\mathrm{b} *}$ & 0.71 & $-0.41^{\mathrm{b}}$ & 0.66 & $0.37^{\mathrm{a}}+$ & $1 \cdot 10$ \\
\hline Fresh fruits without added sugar & $-0.46^{\mathrm{c} *}$ & 0.74 & $-0.25^{\mathrm{b}}$ & 0.81 & $0.35^{\mathrm{a}}+$ & 1.06 \\
\hline Fresh fruits with added sugar & $0.27^{\mathrm{a}}+$ & $1 \cdot 13$ & $-0.13^{b *}$ & 0.79 & $-0.09^{b}$ & 0.98 \\
\hline Water & $0.06^{a}$ & 0.99 & $-0.46^{\mathrm{b} *}$ & 0.96 & $0.15^{\mathrm{a}} \dagger$ & 0.97 \\
\hline Fruit juices & $0.15^{\mathrm{a}} \dagger$ & 1.05 & $0 \cdot 11^{\mathrm{a}}$ & $1 \cdot 15$ & $-0 \cdot 12^{b *}$ & 0.89 \\
\hline Sweetened drinks & $-0.05^{b}$ & 0.73 & $0.68^{\mathrm{a}} \dagger$ & $1 \cdot 71$ & $-0 \cdot 25^{\mathrm{c} *}$ & 0.47 \\
\hline Diet drinks & $-0 \cdot 11^{\mathrm{b}}$ & 0.51 & $0.55^{\mathrm{a}} \dagger$ & 1.92 & $-0 \cdot 16^{\mathrm{b} *}$ & 0.40 \\
\hline Breakfast cereals, muesli, sweetened & $0.31^{a}+$ & $1 \cdot 21$ & $-0.02^{b}$ & 0.87 & $-0 \cdot 16^{\mathrm{c} *}$ & 0.88 \\
\hline Porridge, oat meal, gruel, cereals, muesli, unsweetened & $-0 \cdot 19^{b}$ & 0.77 & $-0.40^{\mathrm{c} *}$ & 0.54 & $0.26^{\mathrm{a}} \dagger$ & $1 \cdot 16$ \\
\hline Plain unsweetened milk & $-0 \cdot 22^{b}$ & 0.85 & $-0.26^{\mathrm{c} *}$ & 0.86 & $0.22^{\mathrm{a}}+$ & 1.07 \\
\hline Sweetened milk & $0.28^{\mathrm{a}} \dagger$ & $1 \cdot 12$ & $-0.05^{\mathrm{b}}$ & 0.95 & $-0 \cdot 13^{\mathrm{C} *}$ & 0.92 \\
\hline Plain unsweetened yogurt or kefir & $0.04^{b}$ & 0.94 & $-0.35^{\mathrm{c} *}$ & 0.61 & $0.12^{\mathrm{a}} \dagger$ & $1 \cdot 12$ \\
\hline Sweet yogurt, fermented milk beverages & $-0.13^{\mathrm{c} *}$ & 0.91 & $0.14^{a}+$ & 1.04 & $0.01^{\mathrm{b}}$ & 1.02 \\
\hline Fresh or frozen fish, not fried & $0.00^{b}$ & 0.96 & $-0.39^{c *}$ & 0.82 & $0.16^{\mathrm{a}}+$ & 1.04 \\
\hline Fried fish, fish fingers & $0.08^{\mathrm{a}} \dagger$ & 1.04 & $-0.04^{b *}$ & 0.89 & $-0.02^{b}$ & 1.02 \\
\hline Cold cuts, preserved, ready-to-cook meat products & $-0.16^{\mathrm{c} *}$ & 0.81 & $0.48^{\mathrm{a}}+$ & $1 \cdot 18$ & $-0 \cdot 11^{\mathrm{b}}$ & 0.95 \\
\hline Fresh meat, not fried & $0.17^{\mathrm{a}}+$ & 0.94 & $-0.23^{\mathrm{c} *}$ & $1 \cdot 08$ & $0.00^{\mathrm{b}}$ & 0.98 \\
\hline Fried meat & $-0.28^{\mathrm{c} \star}$ & 0.74 & $0.59^{\mathrm{a}}+$ & $1 \cdot 23$ & $-0.09^{b}$ & 0.92 \\
\hline Fried or scrambled eggs & $0.30^{\mathrm{a}}+$ & 1.50 & $-0.14^{\mathrm{b} *}$ & 0.69 & $-0 \cdot 10^{b}$ & 0.70 \\
\hline Boiled or poached eggs & $0 \cdot 10^{\mathrm{a}} \dagger$ & $1 \cdot 13$ & $-0.23^{b *}$ & 0.82 & $0.04^{a}$ & 0.98 \\
\hline Mayonnaise, mayonnaise-based products & $0.09^{b}$ & 1.07 & $0.50^{\mathrm{a}}+$ & 1.42 & $-0.25^{\mathrm{c} *}$ & 0.59 \\
\hline Cheese & $0.01^{b}$ & 0.95 & $-0.23^{\mathrm{c} *}$ & 0.96 & $0.09^{\mathrm{a}}+$ & 1.03 \\
\hline Jam, honey & $-0.09^{b *}$ & 0.86 & $0 \cdot 13^{a}+$ & $1 \cdot 10$ & $-0.01^{b}$ & 1.02 \\
\hline Chocolate- or nut-based spreads & $0 \cdot 10^{b}$ & 0.89 & $0.84^{a}+$ & $1 \cdot 38$ & $-0.39^{\mathrm{c} *}$ & 0.55 \\
\hline Butter, margarine on bread & $-0.16^{\mathrm{c} *}$ & 0.76 & $-0.05^{b}$ & 1.03 & $0.11^{a}+$ & 1.08 \\
\hline Reduced-fat products on bread & $-0.23^{c *}$ & 0.59 & $0.05^{\mathrm{b}}$ & 1.04 & $0.10^{\mathrm{a}}+$ & $1 \cdot 13$ \\
\hline Ketchup & $0.37^{\mathrm{a}}+$ & 1.44 & $0.04^{b}$ & 0.85 & $-0 \cdot 21^{c *}$ & 0.65 \\
\hline White bread, white roll, white crispbread & $0.06^{a}+$ & 1.00 & $0.04^{a}$ & 1.01 & $-0.05^{b *}$ & 0.99 \\
\hline Wholemeal bread, dark roll, dark crispbread & $-0.35^{\mathrm{b} *}$ & 0.68 & $0 \cdot 15^{\mathrm{a}}+$ & $1 \cdot 13$ & $0 \cdot 12^{\mathrm{a}}$ & 1.04 \\
\hline Pasta, noodles, rice & $0 \cdot 00^{\mathrm{a}}$ & 1.05 & $-0.21^{\mathrm{b} *}$ & 0.81 & $0.09^{a}+$ & 1.03 \\
\hline Dish of milled cereals & $0.17^{\mathrm{a}}+$ & $1 \cdot 29$ & $-0.23^{c *}$ & 0.55 & $0.01^{\mathrm{b}}$ & 0.95 \\
\hline Pizza as main dish & $0.63^{\mathrm{a}}+$ & 1.49 & $-0.16^{b}$ & 0.68 & $-0 \cdot 27^{\mathrm{c} *}$ & 0.53 \\
\hline Hamburgers, hot dogs, kebabs, wraps, falafel & $0.31^{a}+$ & $1 \cdot 15$ & $-0.36^{c *}$ & 0.70 & $-0.02^{b}$ & 0.97 \\
\hline Nuts, seeds, dried fruits & $0.16^{\mathrm{a}} \dagger$ & $1 \cdot 13$ & $-0.28^{\mathrm{C} *}$ & 0.65 & $0.03^{b}$ & 1.02 \\
\hline Crisps, maize (corn) crisps, popcorn & $0.42^{\mathrm{a}} \dagger$ & $1 \cdot 23$ & $0 \cdot 17^{\mathrm{b}}$ & 1.06 & $-0 \cdot 29^{\mathrm{c} *}$ & 0.70 \\
\hline Savoury pastries, fritters & $0.78^{a}+$ & 1.41 & $-0.31^{\mathrm{b} *}$ & 0.54 & $-0.29^{b}$ & 0.56 \\
\hline Chocolate, candy bars & $0.24^{a}$ & $1 \cdot 12$ & $0.36^{a}+$ & $1 \cdot 25$ & $-0.27^{b *}$ & 0.69 \\
\hline Candies, loose candies, marshmallows & $-0 \cdot 13^{b}$ & 0.78 & $0.78^{\mathrm{a}}+$ & 1.48 & $-0.25^{\mathrm{c} *}$ & 0.64 \\
\hline Biscuits, packaged cakes, pastries, puddings & $-0.07^{b}$ & 0.79 & $0.83^{a}+$ & 1.44 & $-0.30^{c *}$ & 0.63 \\
\hline Ice cream, milk- or fruit-based bars & $0.30^{\mathrm{a}} \dagger$ & $1 \cdot 26$ & $-0.08^{b}$ & 0.95 & $-0.12^{b *}$ & 0.81 \\
\hline
\end{tabular}

compared with younger children. The processed cluster included a lower percentage of normal-weight children and a higher percentage of obese children compared with the other two clusters. The biggest differences were observed between the countries, i.e. certain countries represented up to $46 \%$ of one cluster. Thus, the sweet cluster was mainly represented by Belgian and German children, the processed cluster by Italian, Cypriot, Estonian and Spanish children, while the healthy cluster included a high percentage of Swedish children.

Table 4 summarises the percentage of children being allocated to the same cluster at T0 and T1 and those being allocated to different clusters (see online supplementary Table S1 for the same proportions taking into account only subjects with complete information). With $85 \%$ of the children being allocated to the healthy cluster at both $\mathrm{T} 0$ and $\mathrm{T} 1$, this cluster was the one with the greatest stability. Only $46 \%$ of the children in the processed cluster at T0 remained in this cluster at T1, while $43 \%$ switched to the healthy cluster at T1. Also, 382 children (20\%) allocated to the sweet cluster at T0 changed to the healthy cluster at T1. No differences in the percentage of children allocated to the same or different clusters at T0 and T1 were found between the interventions and the control regions (data not shown).

Table 5 presents OR and 95\% CI for the associations between the identified dietary patterns and socio-economic characteristics. Girls (OR 0.88, 95\% CI 0.79, 0.98) and children with higher-educated fathers (OR $0.73,95 \%$ CI $0.59,0.91$ ) were less likely to be included in the processed cluster at T0 and $\mathrm{T} 1$, while the $\mathrm{OR}$ were higher for older children (OR $1 \cdot 23,95 \% \mathrm{CI} 1 \cdot 10,1 \cdot 38$ ) and migrants (OR $1 \cdot 24,95 \% \mathrm{CI}$ 
Table 3. Description of the included study population, stratified by cluster membership, at baseline (T0) and follow-up (T1) (Number of participants and percentages)

\begin{tabular}{|c|c|c|c|c|c|c|c|c|c|c|c|c|c|c|}
\hline & \multicolumn{4}{|c|}{ Processed } & \multicolumn{4}{|c|}{ Sweet } & \multicolumn{4}{|c|}{ Healthy } & & \\
\hline & \multicolumn{2}{|c|}{ T0 } & \multicolumn{2}{|c|}{$\mathrm{T} 1$} & \multicolumn{2}{|c|}{ T0 } & \multicolumn{2}{|c|}{$\mathrm{T} 1$} & \multicolumn{2}{|c|}{ TO } & \multicolumn{2}{|c|}{$\mathrm{T} 1$} & \multicolumn{2}{|c|}{ Total } \\
\hline & $n$ & $\%$ & $n$ & $\%$ & $n$ & $\%$ & $n$ & $\%$ & $n$ & $\%$ & $n$ & $\%$ & $n$ & $\%$ \\
\hline Total & 4427 & & 2554 & & 1910 & & 1939 & & 2964 & & 4808 & & 9301 & \\
\hline \multicolumn{15}{|l|}{ Sex } \\
\hline Boys & 2240 & 51 & 1342 & 53 & 999 & 52 & 1037 & 54 & 1444 & 49 & 2304 & 48 & 4683 & 51 \\
\hline Girls & 2187 & 49 & 1212 & 47 & 911 & 48 & 902 & 46 & 1520 & 51 & 2504 & 52 & 4618 & 49 \\
\hline \multicolumn{15}{|l|}{ Age } \\
\hline$<6$ years & 1858 & 42 & 1042 & 41 & 895 & 47 & 903 & 47 & 1497 & 51 & 2305 & 48 & 4250 & 46 \\
\hline$\geq 6$ years & 2569 & 58 & 1512 & 59 & 1015 & 53 & 1036 & 53 & 1467 & 49 & 2503 & 52 & 5051 & 54 \\
\hline \multicolumn{15}{|l|}{ BMI status } \\
\hline Underweight & 435 & 10 & 269 & 10 & 251 & 13 & 261 & 14 & 317 & 10 & 473 & 9 & 1003 & 11 \\
\hline Normal weight & 2997 & 68 & 1751 & 69 & 1417 & 74 & 1443 & 74 & 2180 & 74 & 3400 & 71 & 6594 & 71 \\
\hline Overweight & 626 & 14 & 338 & 13 & 166 & 9 & 170 & 9 & 320 & 11 & 604 & 13 & 1112 & 12 \\
\hline Obese & 369 & 8 & 196 & 8 & 166 & 4 & 65 & 3 & 320 & 5 & 331 & 7 & 592 & 6 \\
\hline \multicolumn{15}{|l|}{ Country } \\
\hline Italy & 1032 & 23 & 579 & 23 & 181 & 10 & 221 & 11 & 261 & 9 & 674 & 14 & 1474 & 16 \\
\hline Estonia & 749 & 17 & 393 & 15 & 100 & 5 & 110 & 6 & 397 & 13 & 743 & 16 & 1246 & 13 \\
\hline Cyprus & 795 & 18 & 680 & 27 & 6 & 1 & 8 & 1 & 235 & 8 & 348 & 7 & 1036 & 11 \\
\hline Belgium & 72 & 2 & 43 & 2 & 877 & 46 & 867 & 45 & 141 & 5 & 180 & 4 & 1090 & 12 \\
\hline Sweden & 64 & 1 & 35 & 1 & 34 & 2 & 53 & 3 & 1257 & 42 & 1267 & 26 & 1355 & 15 \\
\hline Germany & 161 & 4 & 98 & 4 & 558 & 29 & 464 & 24 & 259 & 9 & 416 & 9 & 978 & 10 \\
\hline Hungary & 680 & 15 & 328 & 13 & 99 & 5 & 148 & 8 & 207 & 7 & 510 & 11 & 986 & 11 \\
\hline Spain & 874 & 20 & 398 & 16 & 55 & 3 & 68 & 4 & 207 & 7 & 670 & 14 & 1136 & 12 \\
\hline
\end{tabular}

$1 \cdot 05,1 \cdot 46)$ than for younger children and non-migrants. Girls (OR 0.78, 95\% CI 0.66, 0.92), migrants (OR 0.40, 95\% CI $0.31,0.52)$, and children with the highest educated mothers (OR 0.65, 95\% CI 0.47, 0.89) and fathers (OR 0.73, 95\% CI 0.54, 0.99) and highest household income (OR 0.77, 95\% CI $0.61,0.97)$ were less lik ely to be allocated to the sweet cluster at T0 and T1. Obese children (OR 1.37, 95\% CI 1.08, 1.74) and children with higher-educated mothers (OR 1.61, 95\% CI 1.28, 2.04 ) and fathers (OR 1.51, 95\% CI 1.20, 1.90) were more likely to be allocated to the healthy cluster at both time points. Girls (OR 1.16, 95\% CI 1.04, 1.31) and children with the highest household income (OR 1.31, 95\% CI 1.12, 1.53) were also more likely to be allocated to the healthy cluster at T0 and T1. Older children (OR 0.65, 95\% CI 0.58, 0.73) were less likely to be allocated to the healthy cluster. Girls (OR 1.18, 95\% CI 1.07, 1.31), obese children (OR 1.41, 95\% CI $1.12,1.78)$ and children with higher-educated fathers (OR $1.24,95 \%$ CI $1.02,1.50)$ were more likely to change from the processed/sweet cluster at T0 to the healthy cluster at T1. Finally, obese children (OR 0.54, 95\% CI 0.35, 0.85) were less likely to change from the healthy cluster at T0 to the processed/sweet cluster at T1.

\section{Discussion}

The present study derived dietary patterns based on a cluster analysis performed at two different time points in 2- to 9-yearold children participating in the IDEFICS Study. Overall, three consistent dietary patterns were identified at T0 and T1: a processed cluster, showing higher frequencies of consumption of snacks and fast food and lower frequencies of vegetables and wholemeal products; a sweet cluster, showing higher frequencies of consumption of biscuits and sweet products, candies, and sweetened drinks; a healthy cluster, showing higher frequencies of consumption of fruits, vegetables and wholemeal products, and lower frequencies of consumption of processed food products. These three patterns presented similar profiles of relative frequencies of food consumption at each time point, allowing us to assess which children remained in the same patterns and who changed their dietary patterns between T0 and T1. The cluster membership was additionally found to be associated with a number of socioeconomic indicators, namely paternal and maternal education levels, household income and migrant status.

Although dietary patterns are dependent on the population considered and therefore not completely comparable between studies, previous reports extracting dietary patterns of children using cluster analysis found similar results. A British study in children aged 1-4 years has also identified three clusters that were labelled as healthy diet, convenience diet and traditional diet ${ }^{(11)}$. Another recent British study in 7-year-old children has singled out processed, plant-based and traditional

Table 4. Cross-tabulation between the cluster memberships of children at baseline (T0) and follow-up (T1)

(Number of participants and percentages)

\begin{tabular}{|c|c|c|c|c|c|c|c|}
\hline \multirow{3}{*}{$\begin{array}{l}\text { Cluster } \\
\text { membership } \\
\text { at T1 }\end{array}$} & \multicolumn{7}{|c|}{ Cluster membership at T0 } \\
\hline & \multicolumn{2}{|c|}{ Processed } & \multicolumn{2}{|c|}{ Sweet } & \multicolumn{2}{|c|}{ Healthy } & \multirow{2}{*}{$\begin{array}{c}\text { Total } \\
n\end{array}$} \\
\hline & $n$ & $\%$ & $n$ & $\%$ & $n$ & $\%$ & \\
\hline Processed & 2046 & 46 & 228 & 12 & 280 & 9 & 2554 \\
\hline Sweet & 474 & 11 & 1300 & 68 & 165 & 6 & 1939 \\
\hline Healthy & 1907 & 43 & 382 & 20 & 2519 & 85 & 4808 \\
\hline Total & 4427 & & 1910 & & 2964 & & 9301 \\
\hline
\end{tabular}


Table 5. Associations between the cluster memberships over time (each group compared with all the other combinations of cluster memberships) and socio-economic characteristics*

(Odds ratios and $95 \%$ confidence intervals)

\begin{tabular}{|c|c|c|c|c|c|c|c|c|c|c|}
\hline & \multicolumn{2}{|c|}{$\begin{array}{l}\text { Processed cluster } \\
\text { at two time points } \\
\qquad(n 2046)\end{array}$} & \multicolumn{2}{|c|}{$\begin{array}{l}\text { Sweet cluster at } \\
\text { two time points } \\
\quad(n 1300)\end{array}$} & \multicolumn{2}{|c|}{$\begin{array}{l}\text { Healthy cluster at } \\
\text { two time points } \\
\text { (n 2519) }\end{array}$} & \multicolumn{2}{|c|}{$\begin{array}{l}\text { Processed/sweet } \\
\text { cluster at T0, } \\
\text { healthy cluster } \\
\text { at T1 ( } n 2289)\end{array}$} & \multicolumn{2}{|c|}{$\begin{array}{l}\text { Healthy cluster at } \\
\text { T0, processed/ } \\
\text { sweet cluster } \\
\text { at T1 ( } n 445)\end{array}$} \\
\hline & OR & $95 \% \mathrm{Cl}$ & OR & $95 \% \mathrm{Cl}$ & OR & $95 \% \mathrm{Cl}$ & OR & $95 \% \mathrm{Cl}$ & OR & $95 \% \mathrm{Cl}$ \\
\hline \multicolumn{11}{|l|}{ Sex } \\
\hline Boys (n 4683) & 1.00 & & 1.00 & & 1.00 & & 1.00 & & 1.00 & \\
\hline Girls (n 4618) & 0.88 & $0.79,0.98$ & 0.78 & $0.66,0.92$ & $1 \cdot 16$ & $1 \cdot 04,1 \cdot 31$ & $1 \cdot 18$ & $1.07,1.31$ & 1.01 & $0.83,1.22$ \\
\hline \multicolumn{11}{|l|}{ Age group } \\
\hline$<6$ years $(n 4250)$ & 1.00 & & 1.00 & & 1.00 & & 1.00 & & 1.00 & \\
\hline$>6$ years $(n 5051)$ & $1 \cdot 23$ & $1 \cdot 10,1 \cdot 38$ & $1 \cdot 14$ & $0.97,1.35$ & 0.65 & $0.58,0.73$ & 1.00 & $0.90,1.11$ & 1.07 & $0.88,1.30$ \\
\hline \multicolumn{11}{|l|}{ BMI status } \\
\hline Normal weight ( $n$ 6462) & 1.00 & & 1.00 & & 1.00 & & 1.00 & & 1.00 & \\
\hline Overweight ( $n$ 1098) & 0.96 & $0.80,1 \cdot 15$ & 0.79 & $0.62,1.02$ & 1.02 & $0.85,1.23$ & $1 \cdot 25$ & $1.04,1.51$ & 0.96 & $0.71,1.28$ \\
\hline Obese $(n 598)$ & 0.81 & $0.65,1.03$ & 0.81 & $0.57,1.16$ & $1 \cdot 37$ & $1.08,1.74$ & 1.41 & $1 \cdot 12,1 \cdot 78$ & 0.54 & $0.35,0.85$ \\
\hline Missing ( $n$ 1045) & 0.73 & $0.56,0.95$ & 0.49 & $0.30,0.81$ & 1.52 & $1.15,2.03$ & 1.64 & $1 \cdot 27,2 \cdot 13$ & 0.60 & $0.35,1.02$ \\
\hline \multicolumn{11}{|l|}{ Migrant status } \\
\hline Non-migrant ( $n$ 7951) & 1.00 & & 1.00 & & 1.00 & & 1.00 & & 1.00 & \\
\hline Migrant (n 1252) & $1 \cdot 24$ & $1.05,1.46$ & 0.40 & $0.31,0.52$ & 0.98 & $0.82,1.18$ & 1.06 & $0.90,1.23$ & 0.88 & $0.66,1.18$ \\
\hline \multicolumn{11}{|l|}{ Maternal ISCED level } \\
\hline Low (n 1406) & 1.00 & & 1.00 & & 1.00 & & 1.00 & & 1.00 & \\
\hline Medium ( $n$ 4610) & $1 \cdot 15$ & $0.96,1.38$ & 0.80 & $0.63,1.01$ & $1 \cdot 31$ & $1.07,1.60$ & 0.98 & $0 \cdot 84,1 \cdot 15$ & 1.00 & $0.71,1.40$ \\
\hline High (n 2848) & 1.07 & $0.86,1.33$ & 0.65 & $0.47,0.89$ & $1 \cdot 61$ & $1 \cdot 28,2 \cdot 04$ & 0.92 & $0.76,1.12$ & 1.03 & $0.69,1.52$ \\
\hline Missing (n 339) & $1 \cdot 82$ & $1 \cdot 23,2,68$ & 0.83 & $0.56,1.24$ & 0.83 & $0.56,1.22$ & 0.95 & $0.68,1.32$ & 1.07 & $0.59,1.97$ \\
\hline \multicolumn{11}{|l|}{ Paternal ISCED level } \\
\hline Low (n 1247) & 1.00 & & 1.00 & & 1.00 & & 1.00 & & 1.00 & \\
\hline Medium ( $n$ 4814) & 0.84 & $0.71,0.99$ & 0.81 & $0.63,1.04$ & $1 \cdot 22$ & $1.00,1.48$ & $1 \cdot 27$ & $1.09,1.49$ & 1.08 & $0.77,1.40$ \\
\hline High (n 2472) & 0.73 & $0.59,0.91$ & 0.73 & $0.54,0.99$ & 1.51 & $1 \cdot 20,1.90$ & $1 \cdot 24$ & $1.02,1.50$ & $1 \cdot 15$ & $0.78,1.70$ \\
\hline Missing (670) & 0.96 & $0.72,1.29$ & 0.65 & $0.46,0.92$ & $1 \cdot 15$ & $0.85,1.56$ & $1 \cdot 17$ & $0.91,1.52$ & 1.07 & $0.65,1.77$ \\
\hline \multicolumn{11}{|l|}{ Household income } \\
\hline Low (n 2993) & 1.00 & & 1.00 & & 1.00 & & 1.00 & & 1.00 & \\
\hline Medium (n 2297) & 0.98 & $0 \cdot 84,1 \cdot 13$ & 0.86 & $0.68,1.08$ & 1.07 & $0.91,1.26$ & 1.06 & $0.92,1.21$ & 0.79 & $0.61,1.03$ \\
\hline High (n 2934) & 0.90 & $0.78,1.04$ & 0.77 & $0.61,0.97$ & $1 \cdot 31$ & $1.12,1.53$ & $1 \cdot 11$ & $0.97,1.27$ & 0.75 & $0.57,0.97$ \\
\hline Missing (n 979) & 0.85 & $0.70,1.03$ & 0.71 & $0.52,0.98$ & $1 \cdot 24$ & $0.99,1.54$ & $1 \cdot 19$ & $0.99,1.43$ & 0.82 & $0.59,1.14$ \\
\hline
\end{tabular}

TO, baseline; T1, follow-up after the intervention; ISCED, International Standard Classification of Education.

* All the models were adjusted for country and study region (intervention $v$. control) and for all the other factors presented in the table.

British clusters $^{(13)}$. A study among Chinese children aged 6-13 years has also found three clusters: a healthy pattern; a transitive pattern; a Western pattern ${ }^{(28)}$. However, also different numbers of dietary patterns have been described, ranging from two to seven clusters ${ }^{(12,14,29,30-33)}$. The heterogeneity of the reference populations from different countries and continents, the different dietary assessment methods (FFQ $v$. dietary records), the different number and types of food items included and the use of different clustering algorithms (e.g. $K$-means and Ward's method) are likely explanations for the different results. Nevertheless, similar variations in certain patterns have been repeatedly reported across different populations. This is especially true for the patterns labelled as healthy or health-conscious ${ }^{(26)}$.

A previous study has derived four dietary patterns from the IDEFICS baseline data by applying the PCA ${ }^{(20)}$. The first pattern was labelled 'snacking', with the highest loadings for hamburgers, hot dogs, butter, savoury pastries and white bread. The sweet and fat pattern showed the highest loadings for sweet products such as chocolate- or nut-based spreads, cakes, puddings and cookies. The third pattern was labelled 'vegetables and wholemeal', with the highest loadings for vegetables, fruits and wholemeal bread. Finally, the protein and water pattern presented the highest loadings for fish, water, eggs and meat. Our cluster solution presents groupings that are similar to the PCA solution. Nevertheless, it also reflects different aspects and detects a different number of factors/clusters. Other studies exist that have compared dietary patterns obtained by applying the PCA and cluster analysis to the same samples ${ }^{(13,34,35)}$. The results showed a general correlation between the methods, although the two methods describe diet in a different way.

Although it was not the focus of the study, we found a higher percentage of overweight/obese children allocated to the healthy cluster than those allocated to the sweet pattern. The results also showed that obese children were more likely to be allocated to the healthy cluster at both time points. The parents of overweight/obese children might be more prone to under-reporting or providing socially acceptable answers than those of normal-weight children, which is also the case among obese adults. Another plausible explanation is that our dietary instrument, similar to most instruments assessing children's diet, reflects the information provided by proxy reporters (parents) and therefore only 
includes meals under parental control. As a result, this questionnaire might not have been able to adequately capture the consumption of certain high-fat, high-sugar foods, potentially beyond parental control ${ }^{(20)}$.

The present study found that children's membership in a specific cluster was associated with parental education. Specifically, children with higher-educated mothers and fathers were more likely to remain in the healthy cluster at the two time points or to change from the processed/sweet cluster to the healthy one. Notably, the association was found to be stronger for paternal education. Most of the findings in the literature have shown a stronger association with maternal education. Nevertheless, few studies have also described a strong paternal influence on children's dietary intake $^{(36,37)}$, suggesting that higher-educated fathers might communicate beneficial roles and healthy behaviour more clearly than lower-educated fathers. The lack of studies showing paternal influence on children's dietary intake underlines the importance of our findings and the need for further evidence. Previous results from the IDEFICS Study also pointed out the association between parental education and children's food consumption ${ }^{(38)}$. A recent publication describing four clusters (processed, healthy, traditional and packed lunch) at three different time points in a sample of British children has also found an association between a child's cluster membership over time and maternal education level ${ }^{(14)}$. In particular, children with lower-educated mothers were more likely to be allocated to the processed cluster at all time points, while children with higher-educated mothers were more likely to remain in the healthy cluster. The present study also found this association in the case of paternal education. Although similar associations have been reported previously using dietary patterns derived from the PCA ${ }^{(39)}$, the use of cluster analysis to describe dietary patterns over time makes it possible to track which children remain in a specific cluster, thus providing more insight into specific subgroups that consistently show unhealthy dietary patterns.

The present study is subject to a number of limitations. First, the IDEFICS Study was not designed to be nationally representative. Participation in the IDEFICS Study was voluntary, which means that some population groups, e.g. lower-educated or high-income individuals, may have been less willing to take part in the study. The direction of a possible bias cannot be predicted because no systematic information on non-participants is available. Moreover, the direction of the bias usually points in opposite directions for lower and higher SES. A further limitation is the fact that $43 \%$ of the initial baseline cohort did not participate at follow-up and/or did not provide complete data, precluding their inclusion in the present study. Excluded participants showed a higher prevalence of overweight/obesity and a higher percentage of lower-educated parents (see online supplementary Table S2). Consequently, a selection bias cannot be ruled out. Additionally, participants without valid information on maternal education were more likely to be allocated to the processed cluster at both time points, which is another reason why a selection bias cannot be ruled out. The CEHQ-FFQ was not designed to reflect total food intake, but rather to capture information on parent-supervised meals. The number of meals under parental control varied between countries. Sweden, for example, had a higher number of meals and a higher percentage of children eating at school. This might partially explain the differences observed in dietary patterns between countries. However, it was still possible to describe socio-economic differences in dietary patterns, as mainly family socio-economic characteristics influence meals under parental control, as opposed to meals at school.

To the best of our knowledge, this is the first multi-centre European study assessing dietary patterns over time using cluster analysis. The large sample size, the wide variety of dietary habits and cultural backgrounds across eight European countries, and the use of a validated dietary instrument shown to provide reproducible estimates of consumption frequencies are the main strengths of the present study. The use of cluster analysis for deriving dietary patterns at two time points allowed us to identify groups of children with persistently unhealthier dietary profiles and to characterise them according to socio-economic indicators. Healthy eating interventions may benefit from the results of the present study, taking the results into consideration to specifically address groups presenting persistently unhealthier dietary patterns.

\section{Supplementary material}

To view supplementary material for this article, please visit http://dx.doi.org/10.1017/S0007114514003663

\section{Acknowledgements}

The authors gratefully acknowledge the children and parents who participated in the IDEFICS Project. The present study, being part of the IDEFICS Project, was published on behalf of the IDEFICS European Consortium (http://www.idefics.eu). The present study was supported by the European Community's Sixth RTD Framework Programme Contract no. 016181 (FOOD).

The authors' contributions are as follows: J. M. F.-A. carried out the statistical analyses with the help of C. B. and I. P. and drafted the manuscript; K. B. supervised the quality-control study protocol; K. B., W. G., V. K., L. R. and I. P. developed the measurement instruments; A. H., G. B., G. E., I. I., T. V., Y. A. K., E. K., I. H. and L. A. M. supervised the national data collection procedures. All authors read and critically reviewed the manuscript.

None of the authors has any conflicts of interest to declare.

\section{References}

1. Marmot M (2005) Social determinants of health inequalities. Lancet 365, 1099-1104.

2. Mackenbach JP, Stirbu I, Roskam AJ, et al. (2008) Socioeconomic inequalities in health in 22 European countries. $N$ Engl J Med 358, 2468-2481.

3. Nicklas TA, Baranowski T, Cullen KW, et al. (2001) Eating patterns, dietary quality and obesity. J Am Coll Nutr 20, 599-608.

4. Darmon N \& Drewnowski A (2008) Does social class predict diet quality? Am J Clin Nutr 87, 1107-1117. 
5. Van der Horst K, Oenema A, Ferreira I, et al. (2007) A systematic review of environmental correlates of obesity-related dietary behaviors in youth. Health Educ Res 22, 203-226.

6. Sausenthaler S, Kompauer I, Mielck A, et al. (2007) Impact of parental education and income inequality on children's food intake. Public Health Nutr 10, 24-33.

7. Richter A, Heidemann C, Schulze MB, et al. (2012) Dietary patterns of adolescents in Germany - associations with nutrient intake and other health related lifestyle characteristics. BMC Pediatr 12, 35.

8. Craig LC, McNeill G, Macdiarmid JI, et al. (2010) Dietary patterns of school-age children in Scotland: association with socio-economic indicators, physical activity and obesity. BrJ Nutr 103, 319-334.

9. Moreira P, Santos S, Padrao P, et al. (2010) Food patterns according to sociodemographics, physical activity, sleeping and obesity in Portuguese children. Int $J$ Environ Res Public Health 7, 1121-1138.

10. Hu FB (2002) Dietary pattern analysis: a new direction in nutritional epidemiology. Curr Opin Lipidol 13, 3-9.

11. Pryer JA \& Rogers S (2009) Dietary patterns among a national sample of British children aged $11 / 2-41 / 2$ years. Public Health Nutr 12, 957-966.

12. Okubo H, Miyake Y, Sasaki S, et al. (2014) Dietary patterns in infancy and their associations with maternal socio-economic and lifestyle factors among 758 Japanese mother-child pairs: the Osaka Maternal and Child Health Study. Matern Child Nutr 10, 213-225.

13. Smith AD, Emmett PM, Newby PK, et al. (2011) A comparison of dietary patterns derived by cluster and principal components analysis in a UK cohort of children. Eur J Clin Nutr 65, 1102-1109.

14. Northstone K, Smith AD, Newby PK, et al. (2013) Longitudinal comparisons of dietary patterns derived by cluster analysis in 7- to 13-year-old children. Br J Nutr 109, 2050-2058.

15. Ahrens W, Bammann K, Siani A, et al. (2011) The IDEFICS cohort: design, characteristics and participation in the baseline survey. Int J Obes (Lond) 35, Suppl. 1, S3-S15.

16. Bammann K, Peplies J, Sjöström M, et al. (2006) Assessment of diet, physical activity and biological, social and environmental factors in a multi-centre European project on diet- and lifestyle-related disorders in children (IDEFICS). J Public Health 14, 279-289.

17. Bammann K, Gwozdz W, Lanfer A, et al. (2013) Socioeconomic factors and childhood overweight in Europe: results from the multi-centre IDEFICS study. Pediatr Obes $\mathbf{8}, 1-12$.

18. Huybrechts I, Bornhorst C, Pala V, et al. (2011) Evaluation of the Children's Eating Habits Questionnaire used in the IDEFICS study by relating urinary calcium and potassium to milk consumption frequencies among European children. Int J Obes 35, Suppl. 1, S69-S78.

19. Lanfer A, Hebestreit A, Ahrens W, et al. (2011) Reproducibility of food consumption frequencies derived from the Children's Eating Habits Questionnaire used in the IDEFICS study. Int J Obes 35, Suppl. 1, S61-S68.

20. Pala V, Lissner L, Hebestreit A, et al. (2013) Dietary patterns and longitudinal change in body mass in European children: a follow-up study on the IDEFICS multicenter cohort. Eur J Clin Nutr 67, 1042-1049.

21. Graham JW (2009) Missing data analysis: making it work in the real world. Annu Rev Psychol 60, 549-576.
22. UNESCO (2006) ISCED 1997. International Standard Classification of Education [UNE SaCOIfS, editor]. Montreal: UNESCO.

23. OECD (2008) Growing Unequal? Income Distribution and Poverty in OECD Countries. Paris: OECD

24. Stomfai S, Ahrens W, Bammann K, et al. (2011) Intra- and inter-observer reliability in anthropometric measurements in children. Int J Obes 35, Suppl. 1, S45-S51.

25. Cole TJ \& Lobstein T (2012) Extended international (IOTF) body mass index cut-offs for thinness, overweight and obesity. Pediatr Obes 7, 284-294.

26. Newby PK \& Tucker KL (2004) Empirically derived eating patterns using factor or cluster analysis: a review. Nutr Rev 62, 177-203.

27. Everitt B, Landau S, Leese M, et al. (2011) Cluster Analysis, 5th ed. London: Wiley.

28. Shang X, Li Y, Liu A, et al. (2012) Dietary pattern and its association with the prevalence of obesity and related cardiometabolic risk factors among Chinese children. PLOS ONE 7, e43183.

29. Knol LL, Haughton B \& Fitzhugh EC (2005) Dietary patterns of young, low-income US children. J Am Diet Assoc 105, 1765-1773.

30. Rodriguez-Ramirez S, Mundo-Rosas V, Garcia-Guerra A, et al. (2011) Dietary patterns are associated with overweight and obesity in Mexican school-age children. Arch Latinoam Nutr 61, 270-278.

31. Rasanen M, Lehtinen JC, Niinikoski H, et al. (2002) Dietary patterns and nutrient intakes of 7-year-old children taking part in an atherosclerosis prevention project in Finland. J Am Diet Assoc 102, 518-524.

32. Campain AC, Morgan MV, Evans RW, et al. (2003) Sugarstarch combinations in food and the relationship to dental caries in low-risk adolescents. Eur J Oral Sci 111, 316-325.

33. Kim JA, Kim SM, Lee JS, et al. (2007) Dietary patterns and the metabolic syndrome in Korean adolescents: 2001 Korean National Health and Nutrition Survey. Diabetes Care 30, 1904-1905.

34. Reedy J, Wirfalt E, Flood A, et al. (2010) Comparing 3 dietary pattern methods - cluster analysis, factor analysis, and index analysis - with colorectal cancer risk: The NIH-AARP Diet and Health Study. Am J Epidemiol 171, 479-487.

35. Crozier SR, Robinson SM, Borland SE, et al. (2006) Dietary patterns in the Southampton Women's Survey. Eur J Clin Nutr 60, 1391-1399.

36. Lee S \& Reicks M (2003) Environmental and behavioral factors are associated with the calcium intake of low-income adolescent girls. J Am Diet Assoc 103, 1526-1529.

37. Hebestreit A, Keimer KM, Hassel H, et al. (2010) What do children understand? Communicating health behavior in a European multicenter study. J Public Health 18, 391-401.

38. Fernandez-Alvira JM, Mouratidou T, Bammann $\mathrm{K}$, et al. (2013) Parental education and frequency of food consumption in European children: the IDEFICS study. Public Health Nutr 16, 487-498.

39. Northstone K \& Emmett P (2005) Multivariate analysis of diet in children at four and seven years of age and associations with socio-demographic characteristics. Eur J Clin Nutr 59, $751-760$ 